\title{
Docetaxel und Trastuzumab: Eine klinisch bedeutsame Kombination
}

\section{Docetaxel and Trastuzumab: A Combination With Clinical Relevance}

\author{
G. Konecny M. Pegram D. Slamon \\ Division of Hematology-Oncology, University of California, Los Angeles
}

\section{Prognostische Bedeutung von HER-2/neu}

HER-2/neu ist ein epidermaler Wachstumsfaktor-R ezeptor. E ine A mplifikation des HER -2/neu-G ens führt zur Ü berexpression des $\mathrm{R}$ ezeptorproteins. $\mathrm{E}$ ine solche Ü berexpression in Mammakarzinomen korreliert mit einem kürzeren rezidivfreien und $\mathrm{G}$ esamtüberleben. D iese B eobachtung wurde erstmals 1987 von Slamon et al. [1] beschrieben. HE R -2/neu-positive Patientinnen überlebten im M edian 3 Jahre, HER -2/neunegative dagegen im M edian 6-7 Jahre. M ittlerweile ist auch Näheres über die U rsachen der ungünstigeren Prognose bekannt: Die extrem hohe Rezeptorzahl auf der Zelloberfläche - bis zu 2 M illionen R ezeptoren pro Tumorzelle - führt zu einer A ktivierung von Signaltransduktionsketten, die zur Proliferation anregen und eine maligne Transformation begünstigen. A ußerdem, und das scheint ebenfalls von großer Bedeutung zu sein, kommt es über eine A ktivierung der Phosphatidylinositol-3'-kinase und des anti-apoptotischen Schlüsselenzyms Proteinkinase B /A kt auch zu einer E rhöhung der Invasivität und M otilität der Tumorzellen (A bb. 1).

\section{Trastuzumab - Therapieerfahrungen und Wirkungsweise}

Es war das Verdienst von Dennis Slamon, HER -2/neu nicht nur als einen wichtigen pathogenetischen Faktor beim Mammakarzinom identifiziert zu haben, sondern diesen R ezeptor vor allem auch als Ziel für therapeutische $M$ aßnahmen erkannt zu haben. E s gelang schließlich, einen ursprünglich in der $M$ aus induzierten A ntikörper gegen $\mathrm{HER}$-2/neu auf gentechnologischem Wege nahezu vollständig zu humanisieren. D ieser A ntikörper (Trastuzumab, H erceptin ${ }^{\circledR}$ ) enthält lediglich noch $5 \%$ hypervariable Regionen vom Mausantikörper und ist ansonsten identisch mit der menschlichen IgG Struktur, einschließlich des intakten Fc-Rezeptors. D ies gewährleistet die biologische Wirkung beim M enschen, ohne eine Induktion immunologischer Reaktionen gegen diesen A ntikörper auszulösen.
In der M onotherapie führte Trastuzumab zu einer A nsprechrate von $23 \%$ bei unvorbehandelten Patientinnen mit immunhistochemisch HER-2/neu-positivem Mammakarzinom und von $15 \%$ in der Second-line-Therapie bei immunhistochemisch HER -2/neu-positiven Patientinnen. N euere A nalysen belegen deutlich höhere A nsprechraten auf Trastuzumab, wenn die Selektion HER-2/neu positiver Patientinnen mit der Floureszenz-in-Situ-H ybridisierung (FISH) erfolgte. So zeigen unvorbehandelte Patientinnen, bei denen eine A mplifikation des HER-2/neu Genes mit FISH nachgewiesen wurde, eine A nsprechrate von $41 \%$. D iese E rgebnisse sind für eine palliative Situation zwar durchaus positiv, der eigentliche Wert von Trastuzumab liegt jedoch in der Kombination mit einer Chemotherapie. In einer randomisierten klinischen Studie ließ sich durch Hinzunahme von Trastuzumab die R emissionsrate in der First-line-Therapie gegenüber alleiniger Chemotherapie von 32 auf $50 \%$ steigern $(p<0.0001)$, das mediane progressionsfreie Intervall von 4.6 auf 7.4 Monate verlängern $(p<0.0001)$ und die 1-Jahres-Ü berlebensrate von 68 auf $79 \%$ erhöhen $(p=0.008)[2,3]$.

D ie klinische Wirksamkeit von Trastuzumab in Kombination mit Zytostatika wie A Ikylantien oder Platinanaloga lässt sich auf molekularer $E$ bene damit erklären, dass der A ntikörper durch B lockierung der Wachstumsfaktorrezeptoren die D NA Reparaturmechanismen hemmt, die nach zytotoxischer Schädigung der DNA in der Zelle anlaufen. Dadurch wird eine Zytostatikaresistenz verhindert. Diese Wirkungsweise des HER -2/neu-A ntikörpers ließ sich als eine $\mathrm{H}$ emmung der R eparatur von Platin-D NA -A ddukten in vitro eindrucksvoll nachweisen [4]. U ntersuchungen im M aus-M odell mit HE R -2/ neu-überexprimierenden $M$ ammakarzinom-Z elllinien ergaben darüber hinaus, dass die R eihenfolge der Verabreichung von Trastuzumab und Chemotherapie eine bedeutsame R olle für die Wirksamkeit der Kombination spielen kann.

E ine wichtige E rfahrung in den zulassungsrelevanten Studien war die unerwartet hohe Inzidenz einer G rad-3/4-K ardiotoxizität von $16 \%$ bei Kombination von Trastuzumab mit dem A nthrazyklin D oxorubicin.G eringergradige kardialeFunktionsstörungen traten insgesamt bei bis zu $27 \%$ der Patientinnen auf. Wenngleich in reduziertem $\mathrm{M}$ aße war auch im Paclitaxel-

\begin{tabular}{ll}
\hline KARGER & ○ 2000 S. Karger G mbH , Freiburg \\
Fax +497614520714 & A ccessible online at: \\
$\begin{array}{l}\text { E-mail Information@K arger.de } \\
\text { www.karger.com }\end{array}$ & www.karger.com/journals/onk
\end{tabular}

Dr. Gottfried Konecny

Division of $\mathrm{H}$ ematology-O nkology

UCLA School of M edizine

10833 Le Corte A ve.

LosA ngeles, CA 900954-1678 (U SA) 


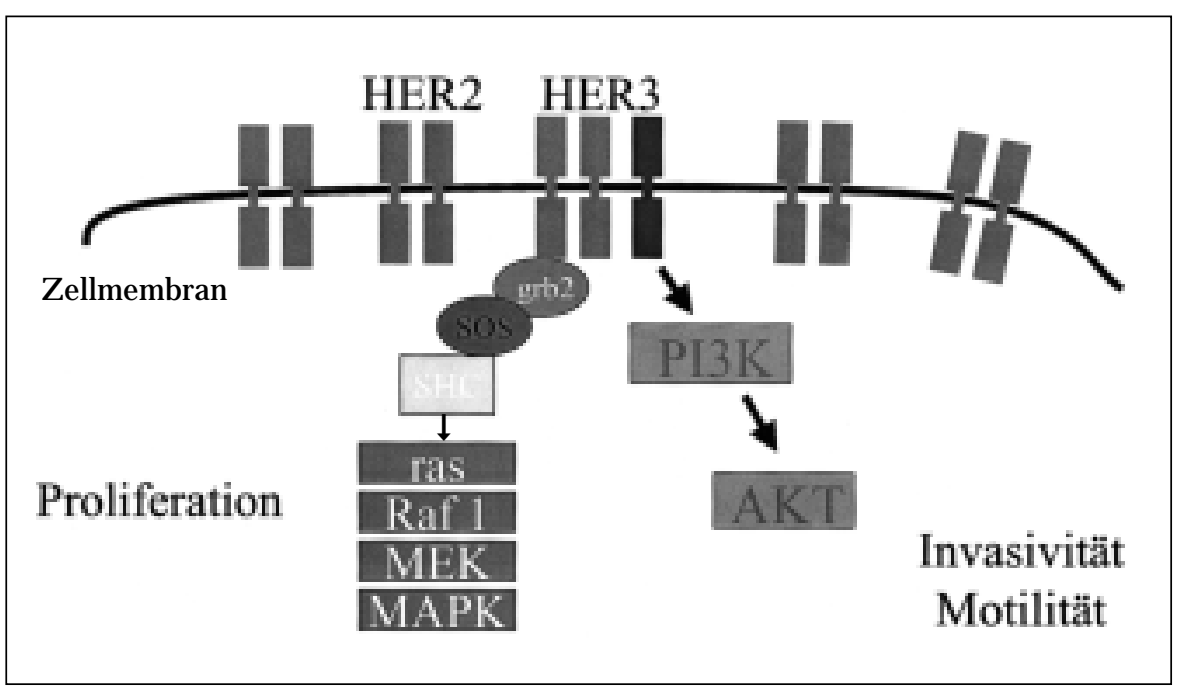

Abb. 1 HE R 2/neu beim M ammakarzinom.

arm eine $K$ ardiotoxizität zu beobachten. D ies wird aber im Wesentlichen darauf zurückgeführt, dass diese Patientinnen in der Regel mit A nthrazyklinen vorbehandelt waren und die kardiotoxische Wechselwirkung mit Trastuzumab offenbar auch in zeitlicher Distanz der beiden Therapien noch zum Tragen kommt.

A us den Ergebnissen dieser klinischen Studie geht deutlich hervor, dass Trastuzumab beim HE R -2/neu-überexprimierenden $\mathrm{M}$ ammakarzinom sinnvollerweise in $\mathrm{K}$ ombination mit einer Chemotherapie eingesetzt werden sollte. In der Therapieplanung sollte aber nach $M$ öglichkeit auf $A$ nthrazykline verzichtet werden. Für zukünftige Studien stellt sich daher die Frage, welches Zytostatikum für eine Kombination mit Trastuzumab am geeignetsten ist. Prinzipiell stehen zahlreiche wirksame Substanzen aus den verschiedenen W irkstoffgruppen zur Verfügung. Wir führten I n-vitro-U ntersuchungen an unterschiedlichen Zelllinien zur wachstumshemmenden Wirkung von Trastuzumab in Verbindung mit einer Reihe von Zytostatika durch. D abei ergaben sich für die Kombinationen von Trastuzumab mit Carboplatin und D ocetaxel jeweils synergistische Wechselwirkungen, mit Paclitaxel und Doxorubicin jeweils additive Interaktionen. Die hohe Wirksamkeit einer Kombination von Trastuzumab mit D ocetaxel und $C$ arboplatin ließ sich in vivo im Mausmodell bestätigen. M ittlerweile sind klinische Studien angelaufen, in denen diese Erkenntnisse B erücksichtigung fanden. So wird Trastuzumab derzeit in mehreren Studien beispielsweise mit D ocetaxel alle 3 Wochen, mit Docetaxel wöchentlich, wie mit Carboplatin/D ocetaxel und Cisplatin/D ocetaxel kombiniert.

\section{Aktuell laufende Studien}

In einer multizentrischen Phase-II-Studie [5] wird D ocetaxel in einer D osierung von $75 \mathrm{mg} / \mathrm{m}^{2}$ alle 3 Wochen mit Trastuzumab (Initialdosis $4 \mathrm{mg} / \mathrm{kg}$, danach $2 \mathrm{mg} / \mathrm{kg}$ wöchentlich) in der First- und Second-line-Therapie von Patientinnen mit HE R -2/neu-positivem metastasierten $M$ ammakarzinom eingesetzt. Trastuzumab wird jeweils zuerst verabreicht, unmittelbar gefolgt von der 1stündigen Docetaxel-Infusion. Das Studienprotokoll sieht die R ekrutierung von 30 Patientinnen vor. D ie Toxizität ist derzeit bei 21 Patientinnen, der Therapieerfolg bei 16 Patientinnen auswertbar. 12 der 16 Patientinnen waren teilweise massiv vorbehandelt, unter anderem auch mit A nthrazyklinen, in drei Fällen mit einer Hochdosistherapie. $B$ isher war keine relevante $K$ ardiotoxizität in dieser Studie zu verzeichnen. Die Behandlung wurde gut toleriert, nur 1 von 21 Patientinnen hatte eine febrile Neutropenie $\mathrm{G}$ rad 3. E ine D ermatitis Grad 2 trat bei 2 Patientinnen, eine des $G$ rades 3 bei 1 Patientin auf. Von den 16 auswertbaren Patientinnen sprachen 7 (44\%) auf die Behandlung an. Die A nsprechrate betrug bei Patientinnen, die im HercepTest ${ }^{\circledR}$ (DA KO) eine starke Ü berexpression des HER-2/neu-R ezeptors zeigten (Score 3+), 55\%, bei Patientinnen mit einer schwächeren Ü berexpression (Score $2+$ ) dagegen nur $20 \%$.

In den U SA wird eine weitere multizentrische Phase-II-Studie mit Trastuzumab und D ocetaxel in der First- oder Second-lineSituation durchgeführt, wobei Docetaxel jedoch in einer Dosierung von $35 \mathrm{mg} / \mathrm{m}^{2}$ wöchentlich verabreicht wird ( N icholson, Thor, G oldstein, G radishar, H avlin, Sledge). Vorgesehen ist eine Rekrutierung von 34 Patientinnen. Auch in dieser Studie fiel die geringe Toxizität auf. Lediglich bei einer von 14 auswertbaren Patientinnen entwickelte sich eine febrile N eutropenie G rad 4, ansonsten traten keine N ebenwirkungen des $\mathrm{G}$ rades 3 oder 4 auf. Insbesondere kam es in keinem Fall zu einer klinisch symptomatischen Verringerung der linksventrikulären E jektionsfraktion. 7 von 13 auswertbaren Patientinnen $(54 \%)$ sprachen auf die B ehandlung an.

$Z$ wei weitere multizentrische Studien rekrutieren derzeit $P$ atientinnen für die First- und Second-line-Therapie des metastasierten $M$ ammakarzinoms mit der Kombination von Trastuzumab, Docetaxel und einem Platin-A nalogon. Für die US-Studie (Slamon, Rivkin, O vermoyer, Carter, Budd, Cobb, A nsari, $\mathrm{G}$ arcia) werden 50 Patientinnen rekrutiert, dabei wird D ocetaxel in einer Dosierung von $75 \mathrm{mg} / \mathrm{m}^{2}$ mit Carboplatin A U C 6 alle 3 Wochen zusammen mit wöchentlichen D osen von Trastuzumab verabreicht. In einer kanadisch-europäischen Studie (Nabholtz, BCIRG Study Group Europe) wird Cisplatin in 
einer D osierung von $75 \mathrm{mg} / \mathrm{m}^{2}$ anstelle von Carboplatin eingesetzt. G eplant ist die R ekrutierung von 40 Patientinnen.

Von den E rgebnissen dieser laufenden Studien beim metastasierten M ammakarzinom wird abhängen, welche therapeutische Strategie am erfolgversprechendsten in die adjuvante Situation übertragen werden kann.

Derzeit sind zwei adjuvante Therapieprotokolle mit Taxanen und Trastuzumab angelaufen. Im nordamerikanischen Protokoll NSA BP B-31 werden Patientinnen in einen der folgenden zwei Studienarme randomisiert:

1. sequenzielle Therapie mit 4 Zyklen D oxorubicin/Cyclophosphamid (AC) und anschließend 4 Zyklen Paclitaxel $175 \mathrm{mg} / \mathrm{m}^{2}$ alle 3 Wochen;

2. wie 1. A rm, jedoch parallel zu Paclitaxel Trastuzumab;

G eplant ist die R ekrutierung von 2700 nodal- und HE R -2/neupositiven Patientinnen. Der Nachweis der Überexpression/ A mplifikation von HER-2/neu erfolgt immunhistochemisch oder mittels Fluoreszenz-in-situ-H ybridisierung (FISH).

D ie zweite, von der Intergroup geplante adjuvante Therapiestudie wurde heftig kritisiert, da das D esign der Studie dem der NSA B P B-31 Studie vergleichbar ist und keinen grundsätzlich neuen Therapiearm enthält. $D$ ie Patientinnen erhalten nach $4 \times A C$, anschließend $90 \mathrm{mg} / \mathrm{m}^{2}$ Paclitaxel wöchentlich über 12 Zyklen. Zusätzlich werden die Patientinnen zwischen der sequenziellen und simultanen Verabreichung von Trastuzumab nach bzw. zusammen mit dem Taxan randomisiert. Es wurde beanstandet, dass diese Fragestellung eine derart umfangreiche Studie mit über 3000 Patientinnen kaum rechtfertigen kann. A uch in dieser Studie wird eine positive Immunhistochemie bzw. FISH als E inschlusskriterium gefordert.

\section{Fazit und Ausblick}

Die bisherigen Erfahrungen mit Trastuzumab in klinischen Studien gaben wichtige $\mathrm{H}$ inweise für die Planung zukünftiger
Studien. Insbesondere müssen L ehren daraus gezogen werden, dass Trastuzumab die Kardiotoxizität der Anthrazykline signifikant erhöht. D ie Substanz sollte daher ausschließlich mit Z ytostatika kombiniert werden, die keine eigene K ardiotoxizität aufweisen. $\mathrm{Zu}$ bevorzugen sind dabei solche Zytostatika, für die in vitro ein Wirkungssynergismus mit Trastuzumab nachzuweisen ist. D as trifft für D ocetaxel ebenso zu wie für die Platinverbindungen. Trastuzumab sollte ferner simultan mit der Chemotherapie angewendet werden, um den Synergismus therapeutisch nutzen zu können. A ufgrund dieser Überlegungen entwickelten $\mathrm{Nabholtz}$ und Slamon das Konzept der BCIR G 006 Studie. Sie umfasst die folgenden 3 Studienarme:

1. sequenzielle Therapie mit 4 Zyklen A C gefolgt von 4 Zyklen D ocetaxel $100 \mathrm{mg} / \mathrm{m}^{2}$ alle 3 Wochen;

2. wie 1. A rm, jedoch parallel zu D ocetaxel Trastuzumab;

3. simultane Therapie mit 6 Zyklen Docetaxel $75 \mathrm{mg} / \mathrm{m}^{2}+$ Carboplatin A U C 6 + Trastuzumab.

Im 2. und 3. Studienarm wird Trastuzumab über die Chemotherapie hinaus für die D auer eines Jahres weiter verabreicht. In dieser Studie repräsentiert der erste Studienarm eine Standardtherapie für M ammakarzinom-Patientinnen mit erhöhtem R ezidivrisiko; der zweite A rm wurde hinzugenommen, um die Vergleichbarkeit mit den angelaufenen amerikanischen Studien zu ermöglichen, und im dritten Studienarm wird die nicht kardiotoxische, innovative Kombinationstherapie untersucht. Wichtige Vorteile dieses Studiendesigns liegen einerseits in dem biologisch fundierten Therapiekonzept unter Ausnutzung des Wirkungssynergismus zwischen Docetaxel bzw. Platinanaloge und Trastuzumab sowie der fehlenden Kardiotoxizität der Therapie. Zudem können zwei D rittel der Patientinnen in dieser Studie von dem absehbaren Nutzen der Trastuzumab-G abe profitieren. Interessant ist zudem, dass der Nachweis der HER -2/neu-Ü berexpression ausschließlich mit FISH, der zur Z eit zuverlässigsten M ethode, geführt wird.

\section{Literatur}

1 Slamon DJ, Clark GM, Wong SG, L evin WJ, U llrich A, M CG uire WL: Human breast cancer: correlation of relapse and survival with amplification of the HER -2/neu oncogene. Science 1987;235:177-182.

2 Slamon D, Leyland-Jones B, Shak S, Paton V, Bajamonde A, Fleming T, Eiermann W, Wolter J, Baselga J, Norton L: A ddition of herceptin (humanized anti-HER 2 antibody) to first-line chemotherapy for HER 2 overexpressing metastatic breast cancer (HER 2+/MBC) markedly increases anticancer ac tivity: A randomized, multinational controlled phase III trial. Proc Am Soc Clin Oncol 1998:17: 377(abstr)

3 Norton L, Slamon D, Leyland-Jones, B, Wolter J, Fleming T, Eiermann W, B aselga J, Mendelsohn J, Bajamonde A, A sh M, Shak S: O verall survival (OS) advantage to simultaneous chemotherapy ( $C R x)$ plus the humanized anti-HER 2 monoclonal antibody Herceptin (H) in HER 2-overexpressing (HER 2+) metastatic breast cancer (M BC). Proc A m Soc Clin O ncol 1999:18:483(abstr).
4 Pietras RJ, Fendly BM, Chazin VR, Pegram MD, Howell SB, Slamon DJ: A ntibody to HER-2/neu receptor blocks DNA repair after cisplatin in human breast and ovarian cancer cells. Oncogene 1994;9: 1829-1838.

$5 \mathrm{E}$ Raefsky, HA Burris, K A lbain, M Huntington, SF Jones, JD Hainsworth, FA Greco, I Erland, $M$ Baskette, A H ussein, CL Vogel: Phase II trial of docetaxel and herceptin as first- or second-line chemotherapy for women with metastatic breast cancer whose tumors overexpress HER 2. Proc A m Soc Clin Oncol 1999;19:523(abstr). 